\title{
EFICIÊNCIA DA SELEÇÃO UNIVARIADA DIRETA E INDIRETA E DE ÍNDICES DE SELEÇÃO EM Eucalyptus grandis ${ }^{1}$
}

\author{
Ildeu Soares Martins ${ }^{2}$, Cosme Damião $\mathrm{Cruz}^{3}$, Adair José Regazzi ${ }^{4}$ e Ismael Eleotério Pires ${ }^{5}$
}

\begin{abstract}
RESUMO - Utilizando experimentos que envolviam famílias de meios-irmãos de Eucalyptus grandis, em seis locais, onde foram anotados dados de circunferência à altura do peito (CAP), altura de plantas (ALT), incidência de ferrugem (FER), incidência de cancro (CAN) e número de árvores normais por parcela (NAR), foram comparadas as metodologias de seleções direta e indireta e índices de seleção (clássico e de Pesek e Baker) com relação às características avaliadas. As seleções direta e indireta não apresentaram distribuição de ganhos genéticos esperados adequada aos propósitos do presente trabalho. O índice clássico e o de Pesek e Baker apresentaram distribuição de ganhos genéticos esperados mais equilibrada.
\end{abstract}

Palavras-chave: Melhoramento genético florestal e índices de seleção.

\section{COMPARISON OF DIRECT AND INDIRECT SELECTION PROCESSES AND SELECTION INDICES IN Eucalyptus grandis}

\begin{abstract}
This study used data from six experiments with half-sib families of Eucalyptus grandis, carried out at six localities, to evaluate the following traits: circumference at breast height (CAP), plant height (ALT), rust incidence (FER), canker incidence (CAN) and number of normal trees per plot (NAR). The direct and indirect selection methodologies were compared with the selection indices (classic and Pesek and Baker method) using all the traits. Neither selection method yielded the expected distribution of genetic gains. Both the classic and Pesek and Baker's indices produced a more suitable distribution of expected genetic gains.
\end{abstract}

Key words: $\quad$ Forest tree improvement and selection indices.

\section{INTRODUÇÃO}

A possibilidade de predição de ganhos a serem obtidos por certa estratégia de melhoramento é uma das mais importantes contribuições da genética quantitativa ao cultivo de plantas. Assim, os processos de seleções direta e indireta surgem como as primeiras alternativas para obtenção de ganhos genéticos compensadores.

Eberhart (1970), Vencovsky (1987) e Vencovsky \& Barriga (1992) apresentaram algoritmos simplificados para obtenção de fórmulas que visam estimativas dos ganhos genéticos. Cruz \& Regazzi (1994) também apresentaram considerações e aplicações sobre diferentes formas de predição dos ganhos de seleção. Os trabalhos de Kageyama (1980, 1983) com Eucalyptus grandis, de Scárdua (1991), Pires (1996) e Paula (1997) com E. camaldulensis e de Xavier (1993) com E. citriodora evidenciaram possibilidades de ganhos genéticos compensadores para algumas características com a seleção entre e dentro de famílias de meios-irmãos, para as referidas espécies.

1 Recebido para publicação em 16.10.2000.

Aceito para publicação em 12.5.2003.

2 Dep. de Engenharia Florestal da Universidade de Brasília - UnB, 70919-970 Brasília-DF. ${ }^{3}$ Dep. de Biologia Geral da Universidade Federal de Viçosa - UFV, 36571-000 Viçosa-MG; ${ }^{4}$ Dep. de Informática da UFV; ${ }^{5}$ Dep. de Engenharia Florestal da UFV. 
Quando a seleção é praticada em determinada característica, normalmente proporciona alterações em outras, em virtude de correlações genéticas. Tal fenômeno é denominado "resposta correlacionada à seleção", e o seu sentido pode ou não ser de interesse do melhorista. A avaliação da magnitude da resposta correlacionada, também denominada resposta indireta, é de particular interesse em caracteres de baixa herdabilidade ou de difícil medição ou identificação (Goldenberg, 1968).

Os métodos até aqui discutidos tratam de seleção univariada, que, na maioria das vezes, não leva a materiais altamente produtivos e adaptados. Assim, deve-se considerar também a seleção para múltiplos caracteres. Para obtenção de materiais genéticos que reúnem uma série de atributos favoráveis e, portanto, mais produtivos e adaptados, uma alternativa seria usar os índices de seleção (Silva, 1982; Cruz \& Regazzi, 1994).

A utilização dos índices de seleção consiste em estabelecer um novo caráter (supercaráter), que é uma combinação linear dos caracteres envolvidos, cujos coeficientes de ponderação são estimados de modo a maximizar a correlação entre o índice e o agregado genotípico (valores genéticos verdadeiros dos candidatos à seleção) (Silva, 1980; White \& Hodge, 1989; Van Vleck, 1993; Cruz \& Regazzi, 1994).

Lin (1978) relatou que a aplicação dos índices de seleção tem limitações, principalmente devido a imprecisões nas matrizes de variâncias e co-variâncias, na mudança dos parâmetros genéticos com a seleção e na estimação dos limites de seleção. White \& Hodge (1989) argumentaram que, devido a alterações nos parâmetros genéticos, provocadas pela seleção com base no índice, é necessária a sua reconstrução a cada ciclo de seleção.

No estabelecimento de pesos econômicos, entretanto, é que se tem verificado a maior limitação da aplicação da teoria do índice clássico (Pesek \& Baker, 1969). BAKER (1986) assegurou que os pesos econômicos devem ser estabelecidos, respeitando-se a proporcionalidade dos caracteres envolvidos.

Cruz (1990) relatou que os pesos econômicos podem ser estabelecidos a partir de estatísticas dos próprios dados experimentais e que o coeficiente de variação genética, por ser diretamente proporcional ao desvio-padrão genético, mantém, de certa forma, a proporcionalidade entre os caracteres e, ainda, por ser adimensional, poderia ser bom referencial no melhoramento genético. Outros critérios de seleção foram desenvolvidos por

R. Árvore, Viçosa-MG, v.27, n.3, p.327-333, 2003 pesquisadores preocupados com as dificuldades e limitações apresentadas pelo índice clássico. Dentre esses critérios, cita-se o índice de Pesek \& Baker (1969), no qual os pesos econômicos são substituídos pelos ganhos desejados pelo melhorista. Nesse método, apesar de os ganhos desejados dispensarem o estabelecimento de pesos econômicos, eles (ganhos desejados) estão sujeitos às limitações impostas pela estrutura genética da população em melhoramento.

Crossbie et al. (1980) e Vieira (1988) sugeriram adotar, como ganhos desejados, o equivalente a uma vez o desvio-padrão genético para cada caráter. Apesar do reconhecimento de certas dificuldades e limitações na utilização da teoria dos índices de seleção, evidenciou-se, de modo geral, que tais índices são vantajosos por proporcionarem maiores ganhos totais, com distribuição, entre os caracteres, mais adequada aos propósitos do melhoramento.

Com relação à utilização da teoria dos índices de seleção no melhoramento florestal, ela ainda é incipiente, principalmente devido ao desconhecimento de técnicas multivariadas e de recursos computacionais para manipulação de grandes volumes de cálculos por parte dos melhoristas florestais. Entretanto, alguns trabalhos têm apontado perspectivas favoráveis (Paula, 1997). O presente trabalho teve por objetivo a comparação da seleção direta e indireta com os índices de seleção, em progênies de Eucalyptus grandis W. Hill ex Maiden.

\section{MATERIAL E MÉTODOS}

\subsection{Caracterização Geral dos Experimentos}

Os experimentos foram instalados e conduzidos em seis locais de experimentação, denominados de TP66A1, TP66A2, TP66B1, TP66B2, TP66C1 e TP66C2, envolvendo famílias de meios-irmãos de E. grandis. Em todos os locais foi utilizado o modelo estatístico em blocos ao acaso, com informação dentro de parcelas, com quatro repetições e seis plantas por parcela, no espaçamento $3 \times 2 \mathrm{~m}$.

O local 1, denominado TP66A1, situa-se no município de Rio Doce, Minas Gerais, na latitude de $19^{\circ} 10^{\prime} \mathrm{S}$, longitude de $42^{\circ} 20^{\prime} \mathrm{W}$ e altitude variando de 220 a $425 \mathrm{~m}$, em situação de encosta. O experimento foi instalado em dezembro de 1993 e a avaliação foi realizada em outubro de 1997, considerando-se os dados de 248 famílias de meios-irmãos. 
O local 2, denominado TP66A2, situa-se também no município de Rio Doce, em condições de baixada. O esperimento foi instalado em dezembro de 1993 e a avaliação foi realizada em maio de 1997, considerando-se os dados de 240 famílias de meios-irmãos.

O local 3, denominado TP66B1, situa-se no município de Sabinópolis, Minas Gerais, na latitude de $18^{\circ} 39^{\prime} \mathrm{S}$, longitude de $42^{\circ} 51^{\prime} \mathrm{W}$ e altitude variando de 820 a $980 \mathrm{~m}$, em situação de encosta. O experimento foi instalado em dezembro de 1993 e a avaliação foi realizada em maio de 1997, considerando-se os dados de 245 famílias de meios-irmãos.

O local 4, denominado TP66B2, situa-se no município de Virginópolis, Minas Gerais, na latitude de $18^{\circ} 45^{\prime} \mathrm{S}$, longitude de $42^{\circ} 34^{\prime} \mathrm{W}$ e altitude variando de 742 a 992 m, em situação de encosta. O experimento foi instalado em dezembro de 1993 e a avaliação foi realizada em março de 1997, considerando-se os dados de 239 famílias de meios-irmãos.

O local 5, denominado TP66C1, situa-se no município de Santa Bárbara, Minas Gerais, na latitude de $19^{\circ} 34^{\prime} \mathrm{S}$, longitude de $42^{\circ} 58^{\prime} \mathrm{W}$ e altitude variando de 815 a $1.099 \mathrm{~m}$, em situação de encosta. O experimento foi instalado em dezembro de 1993 e a avaliação foi realizada em setembro de 1997, considerando-se os dados de 248 famílias de meios-irmãos.

O local 6, denominado TP66C2, situa-se no município de Rio Piracicaba, Minas Gerais, na latitude de $19^{\circ} 27^{\circ} \mathrm{S}$, longitude de $42^{\circ} 52^{\prime} \mathrm{W}$ e altitude variando de 995 a $1.230 \mathrm{~m}$, em situação de encosta. O experimento foi instalado em dezembro de 1993 e a avaliação foi realizada em junho de 1997, considerando-se os dados de 245 famílias de meios-irmãos.

As características avaliadas foram circunferência à altura do peito (CAP) e altura de plantas (ALT), ambas tomadas em nível de plantas dentro de parcelas. Além disto, foram registrados o número de árvores com incidência de ferrugem (FER), número de árvores com incidência de cancro (CAN) e número de árvores normais (NAR) por parcela. Considerou-se normal a árvore viva, inteira e não-bifurcada.

\subsection{Ganhos por Seleções Direta e Indireta}

Foram estimados os ganhos por seleções direta e indireta entre famílias de meios-irmãos para todas as características avaliadas, em todos os locais de experimentação, considerando a seleção de $25 \%$ das melhores famílias. Para as características ALT, CAP e NAR, a seleção foi no sentido de acréscimo e para FER e CAN, no sentido de diminuição.

O ganho por seleção direta foi estimado pela expressão: $\Delta G \%=100\left(D S \times h^{2}\right) / \bar{X}$, em que $\Delta G \%$ é o ganho devido à seleção, expresso em porcentagem da média; DS é o diferencial de seleção; $\mathrm{h}^{2}$ é o coeficiente de herdabilidade; e $\bar{X}$ é a média original.

O ganho por seleção indireta foi estimado pela expressão: $\Delta G X(Y) \%=\left\lfloor D S X(Y) \times h^{2}(X)\right\rfloor / \bar{X}$, em que $\Delta G X(Y)$ é o ganho na característica $X$ (denominada característica secundária), com seleção na característica Y (denominada característica principal); $D S X(Y)$ é o diferencial de seleção indireto na característica $X$, com a seleção em $Y$; e $h^{2}(X)$ é a herdabilidade da característica $X$.

\section{3. Índices de Seleção}

Foram utilizados o índice clássico de Smith e Hazel e o índice com base nos ganhos desejados de Pesek e Baker. Com relação ao índice clássico, proposto por Smith (1936) e Hazel (1943), o índice (I) e o agregado genotípico $(\mathrm{H})$ são assim descritos:

$$
\begin{aligned}
& I=b_{1} x_{1}+b_{2} x_{2}+\ldots \ldots \ldots . . .+b_{n} x_{n}=\sum_{i=1}^{n} b_{i} x_{i}=b^{\prime} x \\
& H=a_{1} g_{1}+a_{2} g_{2}+\ldots \ldots \ldots+a_{n} g_{n}=\sum_{i=1}^{n} a_{i} g_{i}=a^{\prime} g
\end{aligned}
$$

em que n é o número de caracteres avaliados; $b$ ' é um vetor $1 \times \mathrm{n}$ dos coeficientes lineares a serem estimados; $x$ é um vetor $\mathrm{n} \times 1$ de médias dos caracteres; $a^{\prime}$ é um vetor $1 \times n$ de pesos econômicos previamente estabelecidos; e $g$ é um vetor $n \times 1$ de valores genéticos desconhecidos dos $n$ caracteres.

Em função das estruturas do índice e do agregado genotípico, pode-se deduzir que

$$
\hat{b}=P^{-1} G a
$$

em que $P$ é a matriz de co-variâncias fenotípicas e $G$ é a matriz de co-variâncias genéticas entre os caracteres.

$\mathrm{Na}$ aplicação do índice clássico, consideraram-se como pesos econômicos os coeficientes de variação genética de cada caráter, conforme sugestão de Cruz (1990), e ainda outras combinações de pesos econômicos,

R. Árvore, Viçosa-MG, v.27, n.3, p.327-333, 2003 
visando distribuição de ganhos convenientes, de acordo com os propósitos do presente trabalho.

Para construção do índice de Pesek e Baker, devese considerar a expressão do ganho desejado para os vários caracteres, definida por

$$
\Delta G d=\frac{G \hat{b}}{\hat{\sigma}_{I}}
$$

em que $\Delta G d$ é o vetor de ganhos desejados, $G$ é a matriz de variâncias e co-variâncias genéticas entre os caracteres, $\hat{b}$ é o vetor $n \times 1$ de coeficientes do índice, e $\hat{\sigma}_{I}$ é a raiz quadrada da variância do índice. Como $\hat{\sigma}_{I}$ é escalar e não afeta a proporcionalidade do índice, ele pode ser desprezado, obtendo-se

$$
\hat{b}=G^{-1} \Delta G d
$$

Assim, o índice seria estabelecido apenas com a definição do vetor de ganhos desejados e da matriz de variâncias e co-variâncias genotípicas.

Apesar de o referido método dispensar o estabelecimento dos pesos econômicos, estes podem ser estimados, posteriormente, pela expressão

$$
\hat{a}=G^{-1} P \hat{b}
$$

em que $\hat{a}$ é o vetor $n \times 1$ de pesos econômicos estimados e $P$ é a matriz de variâncias e co-variâncias fenotípicas entre os caracteres.

Para construção do índice de Pesek e Baker, consideraram-se como pesos econômicos o equivalente a um desvio-padrão genético para cada caráter, conforme sugestão de Crossbie et al. (1980) e Vieira (1988), e também outras combinações de pesos, visando melhores distribuições de ganhos de acordo com os propósitos do presente trabalho.

Todos os procedimentos estatísticos utilizados foram processados, utilizando-se o aplicativo computacional "GENES FOR WINDOWS" (Cruz, 1998).

\section{RESULTADOS E DISCUSSÃO}

\subsection{Seleções Direta e Indireta entre Médias de Famílias}

As seleções direta e indireta não proporcionaram resultados conjuntos satisfatórios em ganhos nas cinco características avaliadas, uma vez que o interesse era

R. Árvore, Viçosa-MG, v.27, n.3, p.327-333, 2003 obter acréscimo nas características CAP, ALT e NAR e decréscimo em FER e CAN. Essa combinação de resultados não foi atingida em nenhum dos locais, considerando-se a seleção em qualquer uma das características referidas (Quadros de 1 a 6).

No local 1, a seleção nas características de crescimento da planta proporcionou resposta correlacionada entre elas. A seleção em CAP proporcionou resposta favorável em ALT, e vice-versa. Este fato foi atribuído à alta correlação genética entre essas características. Entretanto, essa provocou alterações indesejáveis nas características de sanidade das plantas. A seleção de plantas com maiores CAP e ALT resultou em genótipos mais suscetíveis à ferrugem (FER) e ao cancro (CAN).

A escolha de genótipos com maiores CAP e ALT (implicando maiores volumes de madeiras), simultaneamente com menores incidências de ferrugem e cancro, é de extrema importância para a cultura do eucalipto, uma vez que os controles dessas enfermidades são feitos, utilizando-se de genótipos resistentes ou tolerantes para plantio no campo.

A seleção com base em NAR levou a estimativas de ganhos expressivos em CAP e ALT, mas, também, a respostas em sentido indesejável em FER e CAN. Portanto, quando a seleção é praticada em uma característica cujo interesse era o deslocamento positivo da média, obtêm-se ganhos positivos em todas as outras, e viceversa. Desta forma, é necessária a adoção de metodologias que possam proporcionar combinações de ganhos esperados favoráveis, de acordo com o presente propósito. Nesse contexto, a metodologia multivariada, denominada índices de seleção, pode ser uma alternativa. Nos demais locais de experimentação, resultados semelhantes foram encontrados.

\section{2. Índices de Seleção}

Os índices foram construídos para cada local de experimentação. No local 2, para construção do índice de Pesek e Baker, utilizando como vetor de ganhos desejados o equivalente a um desvio-padrão genético, não se considerou a característica CAN.

Os índices de seleção, construídos com os pesos econômicos referidos (Quadro 7), não proporcionaram distribuição de ganhos condizentes com os propósitos do presente trabalho. Esses ganhos deveriam ser em sentido positivo para as características CAP, ALT e NAR 
Quadro 1 - Respostas esperadas às seleções direta e indireta entre famílias de Eucalyptus grandis no local 1

Table 1 - Expected genetic gains through direct and indirect selections among Eucalyptus grandis families in location 1

\begin{tabular}{|l|r|r|r|r|r|}
\hline \multirow{2}{*}{ Seleção em } & \multicolumn{5}{|c|}{ Resposta Esperada em (\%) } \\
\cline { 2 - 6 } & CAP & \multicolumn{1}{|c|}{ ALT } & FER & \multicolumn{1}{c|}{ CAN } & \multicolumn{1}{c|}{ NAR } \\
\hline CAP & 38,34 & 38,12 & 34,45 & 32,31 & 35,96 \\
ALT & 38,04 & 38,13 & 34,05 & 32,05 & 35,92 \\
FER & $-36,00$ & $-35,65$ & $-33,83$ & $-30,78$ & $-33,02$ \\
CAN & $-34,35$ & $-34,14$ & $-31,31$ & $-31,12$ & $-31,87$ \\
NAR & 36,37 & 36,40 & 31,96 & 30,32 & 34,99 \\
\hline
\end{tabular}

Quadro 2 - Respostas esperadas às seleções direta e indireta entre famílias de Eucalyptus grandis no local 2 Table 2 - Expected genetic gains through direct and indirect selections among Eucalyptus grandis families in location 2

\begin{tabular}{|l|c|c|c|c|c|}
\hline \multirow{2}{*}{ Seleção em } & \multicolumn{5}{|c|}{ Resposta Esperada em (\%) } \\
\cline { 2 - 6 } & CAP & ALT & FER & \multicolumn{1}{c|}{ CAN } & \multicolumn{1}{c|}{ NAR } \\
\hline CAP & 26,84 & 27,07 & 20,07 & 22,74 & 23,83 \\
ALT & 26,43 & 26,82 & 19,76 & 22,46 & 23,81 \\
FER & $-22,79$ & $-22,99$ & $-18,71$ & $-19,95$ & $-20,65$ \\
CAN & $-24,18$ & $-24,47$ & $-18,68$ & $-21,97$ & $-21,86$ \\
NAR & 23,63 & 24,18 & 18,03 & 20,39 & 22,27 \\
\hline
\end{tabular}

Quadro3 - Respostas esperadas às seleções direta e indireta entre famílias de Eucalyptus grandis no local 3

Table 3 - Expected genetic gains through direct and indirect selections among Eucalyptus grandis families in location 3

\begin{tabular}{|l|r|r|r|r|r|}
\hline \multirow{2}{*}{ Seleção em } & \multicolumn{5}{|c|}{ Resposta Esperada em (\%) } \\
\cline { 2 - 6 } & CAP & \multicolumn{1}{|c|}{ ALT } & FER & \multicolumn{1}{c|}{ CAN } & \multicolumn{1}{c|}{ NAR } \\
\hline CAP & 14,31 & 13,53 & 10,83 & 10,61 & 10,94 \\
ALT & 13,90 & 13,66 & 11,09 & 10,82 & 11,19 \\
FER & $-12,51$ & $-12,47$ & $-11,45$ & $-11,13$ & $-11,55$ \\
CAN & $-12,40$ & $-12,31$ & $-11,25$ & $-10,98$ & $-11,35$ \\
NAR & 12,07 & 12,02 & 11,03 & 10,71 & 11,62 \\
\hline
\end{tabular}

e negativo, para FER e CAN. Porém, em alguns casos, os índices obtidos dessa forma conseguiram se aproximar da distribuição de ganhos de interesse, o que é encorajador no sentido de tentar utilizar outros vetores de pesos econômicos, com o intuito de atingir os objetivos almejados.

No local 3, o índice de Pesek e Baker, apesar das baixas magnitudes de ganhos esperados, proporcionou
Quadro 4 - Respostas esperadas às seleções direta e indireta entre famílias de Eucalyptus grandis no local 4 Table 4 - Expected genetic gains through direct and indirect selections among Eucalyptus grandis families in location 4

\begin{tabular}{|c|r|r|r|r|r|}
\hline \multirow{2}{*}{ Seleção em } & \multicolumn{5}{|c|}{ Resposta Esperada em (\%) } \\
\cline { 2 - 6 } & \multicolumn{1}{|c|}{ CAP } & \multicolumn{1}{c|}{ ALT } & \multicolumn{1}{c|}{ FER } & \multicolumn{1}{c|}{ CAN } & \multicolumn{1}{c|}{ NAR } \\
\hline CAP & 14,41 & 13,41 & 8,94 & 10,37 & 10,36 \\
ALT & 13,73 & 12,95 & 8,50 & 10,00 & 10,17 \\
FER & $-9,38$ & $-8,72$ & $-9,20$ & $-7,44$ & $-7,31$ \\
CAN & $-12,03$ & $-11,33$ & $-8,22$ & $-9,29$ & $-9,40$ \\
NAR & 11,67 & 11,19 & 7,85 & 9,13 & 9,47 \\
\hline
\end{tabular}

Quadro 5 - Respostas esperadas às seleções direta e indireta entre famílias de Eucalyptus grandis no local 5 Table 5 - Expected genetic gains through the direct and indirect selections among Eucalyptus grandis families in location 5

\begin{tabular}{|l|r|r|r|r|r|}
\hline \multirow{2}{*}{ Seleção em } & \multicolumn{5}{|c|}{ Resposta Esperada em (\%) } \\
\cline { 2 - 6 } & CAP & \multicolumn{1}{|c|}{ ALT } & \multicolumn{1}{c|}{ FER } & \multicolumn{1}{c|}{ CAN } & \multicolumn{1}{c|}{ NAR } \\
\hline CAP & 22,23 & 21,56 & 16,88 & 17,95 & 16,29 \\
ALT & 22,01 & 21,60 & 17,02 & 18,01 & 16,58 \\
FER & $-17,18$ & $-16,97$ & $-17,74$ & $-14,44$ & $-12,82$ \\
CAN & $-21,02$ & $-20,66$ & $-16,61$ & $-17,53$ & $-15,55$ \\
NAR & 18,67 & 18,62 & 14,44 & 15,22 & 14,58 \\
\hline
\end{tabular}

Quadro 6 - Respostas esperadas às seleções direta e indireta entre famílias Eucalyptus grandis no local 6

Table 6 - Expected genetic gains through direct and indirect selections among Eucalyptus grandis families in location 6

\begin{tabular}{|c|r|r|r|r|r|}
\hline \multirow{2}{*}{ Seleção em } & \multicolumn{5}{|c|}{ Resposta Esperada em (\%) } \\
\cline { 2 - 6 } & \multicolumn{1}{|c|}{ CAP } & \multicolumn{1}{c|}{ ALT } & \multicolumn{1}{c|}{ FER } & \multicolumn{1}{c|}{ CAN } & \multicolumn{1}{c|}{ NAR } \\
\hline CAP & 13,64 & 11,93 & 6,21 & 6,24 & 7,55 \\
ALT & 12,64 & 11,52 & 6,67 & 6,69 & 7,91 \\
FER & $-8,65$ & $-8,76$ & $-7,23$ & $-7,23$ & $-8,10$ \\
CAN & $-8,68$ & $-8,78$ & $-7,22$ & $-7,25$ & $-8,12$ \\
NAR & 9,04 & 8,94 & 6,96 & 6,99 & 8,37 \\
\hline
\end{tabular}

sentidos de ganhos favoráveis para todos os caracteres avaliados (Quadro 7).

Com base nessas considerações, foram propostos outros vetores de pesos econômicos (no caso do índice clássico) ou de ganhos desejados (no caso do índice de Pesek e Baker), para cada local de experimentação, exceto o local 3 (Quadro 8), visando conseguir a melhor distribuição de ganhos esperados, com base nos objetivos

R. Árvore, Viçosa-MG, v.27, n.3, p.327-333, 2003 
do presente trabalho. Na construção desses índices, como as características FER e CAN apresentaram estimativas muito altas de coeficiente de correlação genética, optouse por excluir a segunda delas.

$\mathrm{Na}$ construção de cada um dos índices, os pesos econômicos foram baseados nos parâmetros estimados com os próprios dados experimentais, como os coeficientes de variação genética para os índices clássicos e os desvios-padrão genéticos para o índice de Pesek e Baker. Os índices de seleção, de modo geral, foram vantajosos em relação aos processos de seleções direta e indireta, uma vez que possibilitaram, para todos os locais, ganhos nos sentidos desejados.

Quadro 7 - Respostas esperadas, com a seleção nos índices de Smith e Hazel e de Pesek e Baker, nos seis locais de experimentação

Table 7 - Expected genetic gains with the selection on Smith and Hazel's and Pesek and Baker's indices, in six locations

\begin{tabular}{|l|r|r|r|r|r|}
\hline \multirow{2}{*}{ Índice } & \multicolumn{5}{|c|}{ Resposta Esperada em (\%) } \\
\cline { 2 - 6 } & \multicolumn{1}{|c|}{ CAP } & \multicolumn{1}{|c|}{ ALT } & \multicolumn{1}{c|}{ FER } & CAN & NAR \\
\hline SH1 & 39,62 & 39,46 & 32,14 & 28,59 & 34,18 \\
PB1 & 4,75 & 2,40 & $-4,11$ & $-0,66$ & $-3,73$ \\
SH2 & 26,04 & 26,22 & 14,73 & 18,65 & 19,01 \\
PB2 & 0,30 & $-1,53$ & $-6,26$ & $-3,41$ & $-0,44$ \\
SH3 & 12,34 & 11,11 & 7,6 & 7,45 & 7,35 \\
PB3 & 1,90 & 1,84 & $-1,48$ & $-1,32$ & 0,52 \\
SH4 & 14,00 & 11,74 & 5,32 & 7,37 & 6,94 \\
PB4 & 3,50 & 1,70 & $-2,57$ & 0,66 & 1,09 \\
SH5 & 20,06 & 19,88 & 12,32 & 14,90 & 11,08 \\
PB5 & $-12,04$ & $-12,02$ & $-15,89$ & $-11,72$ & $-9,31$ \\
SH6 & 12,40 & 9,48 & 2,89 & 2,88 & 3,52 \\
PB6 & $-3,10$ & $-1,08$ & $-2,32$ & $-2,27$ & $-1,07$ \\
\hline
\end{tabular}

Shi = índice de Smith e Hazel, com pesos econômicos iguais a um coeficiente de variação genética para cada característica, no local i.

$\mathrm{Pbi}=$ índice de Pesek e Baker, com pesos econômicos iguais a um desvio padrão genético para cada característica, no local i.
O índice de Pesek e Baker foi mais eficiente nos locais 1 e 3 , ao passo que o clássico foi melhor nos demais locais. Assim, não foi identificado um índice adequado para todos os locais, o que indica que em futuros trabalhos com esta espécie, em quaisquer locais de experimentação, deverão ser verificadas as possibilidades de uso de um ou de outro índice, visando as melhores distribuições de ganhos esperados, de acordo com interesses específicos.

\section{CONCLUSÕES}

Os processos de seleção direta e indireta entre médias de famílias não são eficientes no sentido de proporcionar uma distribuição de ganhos esperados adequada aos propósitos do presente trabalho (aumento em CAP, ALT e NAR e redução em FER e CAN, em todos os locais de experimentação).

Os índices de seleção foram mais eficientes no sentido de proporcionar uma distribuição de ganhos esperados mais equilibrada, em todos os locais de experimentação.

\section{REFERÊNCIAS BIBLIOGRÁFICAS}

BAKER, R. J. Selection indices in plant breeding. Florida: CRC Press, 1986. 218 p.

CASTOLDI, F. L. Comparação de métodos multivariados aplicados na seleção em milho. 1987. 118 f. Tese (Doutorado em Genética e Melhoramento) - Universidade Federal de Viçosa, Viçosa, 1997.

CROSSBIE, T. M.; MOCK, J. J.; SMITH, D. S.

Comparison of gains predicted by several selections methods for cold tolerance traits for two maize populations.

Crop Science, v. 20, p. 649-655, 1980.

Quadro 8 - Ganhos genéticos esperados em Eucalyptus grandis, em cada local, com aplicação de seleção usando os pesos econômicos respectivamente para CAP, ALT, FER e NAR

Table 8 - Expected genetic gains in Eucalyptus grandis, using other economic weights

\begin{tabular}{|c|c|c|c|c|c|c|}
\hline \multirow{2}{*}{ Local } & \multirow{2}{*}{ Método (pesos econômicos) } & \multicolumn{5}{|c|}{ Ganhos Esperados (\%) em } \\
\hline & & CAP & ALT & FER & CAN & NAR \\
\hline 1 & Pesek \& Baker $(6: 2,5:-1: 0,5)$ & 5,18 & 2,86 & $-5,47$ & $-0,84$ & 0,69 \\
\hline 2 & Clássico (1: 1: $-34,5: 1$ & 3,41 & 4,02 & $-4,74$ & $-1,41$ & 1,25 \\
\hline 4 & Clásico $(2: 2:-56: 4,4)$ & 4,80 & 4,61 & $-2,21$ & 2,23 & 2,38 \\
\hline 5 & Clássico (1: 1: $-40: 1)$ & 11,72 & 10,98 & $-1,05$ & 7,07 & 6,95 \\
\hline 6 & Clássico (1: 2: -66: 11) & 8,07 & 4,41 & $-0,20$ & $-0,21$ & 0,10 \\
\hline
\end{tabular}

R. Árvore, Viçosa-MG, v.27, n.3, p.327-333, 2003 
CRUZ, C. D. Aplicações de algumas técnicas multivariadas no melhoramento de plantas. 1990. $188 \mathrm{f}$. Tese (Doutorado em Genética e Melhoramento) - Escola Superior de Agricultura "Luis de Queiroz", Piracicaba, 1990.

CRUZ, C. D. Programa GENES-Aplicativo computacional em estatística aplicada à genética. Genetics and Molecular Biology, v. 21, n. 1, p. 135-138, 1998.

CRUZ, C. D.; REGAZZI, A. J. Modelos biométricos aplicados ao melhoramento genético. Viçosa: UFV, Imprensa Universitária, 1994. 390 p.

EBERHART, S. A. Factors affecting efficiencies of breeding methods. African Soils, v. 5, p. 669-672, 1970.

GOLDENBERG, J. B. El empleo de la correlación en el mejoramento genético de las plantas. Fitotecnia Latinoamericana, v. 5, n. 2, p. 1-8, 1968.

HAZEL, L. N. The genetic basis for constructing selection indices. Genetics, v. 28, n. 6, p. 476-490, 1943.

KAGEYAMA, P. Y. Variações genéticas em progênies de uma população de Eucalyptus grandis W. Hill ex Maiden. 1980. $125 \mathrm{f}$. Tese (Doutorado em Genética e Melhoramento) - Escola Superior de Agricultura "Luís de Queiroz", Piracicaba, 1980.

KAGEYAMA, P. Y. Seleção precoce a diferentes idades em progênies jovens de Eucalyptus grandis W. Hill ex Maiden. 1983. 147 f. Tese (Livre Docência) - Escola Superior de Agricultura "Luís de Queiroz", Piracicaba, 1983.

LIN, C. Y. Index selection for genetic improvement of quantitative characters. Theorethacal Applied Genetics, v. 52, p. 49-56, 1978.

PAULA, R. C. Avaliação de diferentes critérios de seleção aplicados em melhoramento florestal. 1997. $74 \mathrm{f}$. Tese (Doutorado em Ciências Florestais) - Universidade Federal de Viçosa, Viçosa, 1997.

PESEK, J.; BAKER, R. J. Desired improvement in relation to selected indices. Canadian Journal Plant Science, v. 49, p. 803-804, 1969.
PIRES, I. E. Eficiência da seleção combinada no melhoramento genético de Eucalyptus spp. 1996. $116 \mathrm{f}$. Tese (Doutorado em Genética e Melhoramento) Universidade Federal de Viçosa, Viçosa, 1996.

SCÁRDUA, F. P. Variabilidade genética em progênies de Eucalyptus camaldulensis Dehnh., na Fazenda Água Limpa - DF, a diferentes idades. 1991. 72 f. Dissertação (Graduação em Engenharia Florestal) - Universidade de Brasília, Brasília, 1991.

SILVA, M. A. Melhoramento animal (índices de seleção). Viçosa, MG: UFV, Imprensa Universitária, 1980. 65 p.

SILVA, M. A. Melhoramento animal (métodos de seleção). Viçosa, MG: UFV, Imprensa Universitária, 1982. $51 \mathrm{p}$.

SMITH. H. F. A discriminant function for plant selection. Ann. Eugenics, v. 7, p. 240-250, 1936.

VAN VLECK, L. D. Selection index and introduction to mixed model methods for genetic improvement of animals: The green book. Boca Raton: CCR Press, 1993. $481 \mathrm{p}$.

VENCOVSKY, R. Herança quantitativa. In: PATERNIANI, E.; VIEGAS, G. P. (Eds). Melhoramento e produção do milho. Campinas: Fundação Cargill, 1987. p.137-214.

VENCOVSKY, R.; BARRIGA, P. Genética biométrica no fitomelhoramento. Ribeirão Preto: Sociedade Brasileira de Genética, 1992. 406 p.

VIEIRA, J. V. Herdabilidade, correlações genéticas e índices de seleção em uma população de cenoura (Daucus carota L.). 1988. 86 f. Tese (Doutorado em Genética e Melhoramento) - Universidade Federal de Viçosa, Viçosa, 1988.

XAVIER, A. Variabilidade genética de óleo essencial e de crescimento em progênies de meios-irmãos de Eucalyptus citriodora Hook. 1993. 72 f. Tese (Mestrado em Ciências Florestais) - Universidade Federa de Viçosa, Viçosa, 1993.

WHITE, T. L.; HODGE, G. R. Predicting breeding values: with applications in forest tree improvement. London: Kluwer Academic Publishers, 1989. 367 p. 